\title{
The influence of knee rigidity on balance corrections: a comparison with responses of cerebellar ataxia patients
}

\author{
L. B. Oude Nijhuis $\cdot$ J. Hegeman $\cdot$ M. Bakker $\cdot$ \\ M. Van Meel • B. R. Bloem · J. H. J. Allum
}

Received: 15 August 2007 / Accepted: 16 January 2008 / Published online: 5 February 2008

(C) Springer-Verlag 2008

\begin{abstract}
Knee rigidity due to aging or disease is associated with falls. A causal relationship between instability and knee rigidity has not been established. Here, we examined whether insufficient knee movement due to knee rigidity could underlie poor balance control in patients. We addressed this by examining the effect of artificially "locking" the knees on balance control in 18 healthy subjects, tested with and without individually fitted knee casts on both legs. Subjects were exposed to sudden rotations of a support surface in six different directions. The primary outcome measure was body centre of mass (COM) movement, and secondary outcome measures included biomechanical responses of the legs, pelvis and trunk. Knee casts caused increased backward COM movement for backward perturbations and decreased vertical COM movement for forward perturbations, and caused little change in lateral COM movement. At the ankles, dorsiflexion was reduced for backward perturbations. With knee casts, there was less uphill hip flexion and more downhill hip flexion. A major difference with knee casts was a reversed pelvis pitch movement and an increased forward trunk motion. These alterations in pitch movement strategies and COM displacements were similar to those we have observed previously in patients with knee rigidity, specifically those with spinocerebellar ataxia (SCA). Pelvis roll and uphill arm
\end{abstract}

L. B. Oude Nijhuis · J. Hegeman · M. Bakker · M. Van Meel ·

J. H. J. Allum ( $\square)$

Division of Audiology and Neurootology,

Department of ORL, University Hospital,

Hebelstrasse 10, 4031 Basel, Switzerland

e-mail: jallum@uhbs.ch

L. B. Oude Nijhuis · M. Bakker · B. R. Bloem

Department of Neurology,

Radboud University Nijmegen Medical Centre,

PO Box 9101, 6500 Nijmegen, The Netherlands abduction were also increased with the casts. This roll movement strategy and minor changes in lateral COM movement were not similar to observations in patients. We conclude that artificial knee rigidity increases instability, as reflected by greater posterior COM displacement following support surface tilts. Healthy controls with knee casts used a pitch movement strategy similar to that of SCA patients to offset their lack of knee movement in regaining balance following multidirectional perturbations. This similarity suggests that reduced knee movements due to knee rigidity may contribute to sagittal plane postural instability in SCA patients and possibly in other patient groups. However in the roll plane, healthy controls rapidly compensate by adjusting arm movements and hip flexion to offset the effects of knee rigidity.

Keywords Knee movements - Balance corrections . Multi-directional perturbations $\cdot$ Knee $\cdot$ Rigidity .

Cerebellar ataxia $\cdot$ Balance control

\section{Introduction}

The human body during quiet stance and after small perturbations of upright stance is often pictured as moving like an inverted pendulum, with rotations about the ankle as the most important means of controlling balance (Nashner and McCollum 1985; Fitzpatrick et al. 1992,1994; Gatev et al. 1999). Another recovery strategy, the hip strategy, is characterised by anti-phasic ankle and hip rotations, and is used to describe movements following perturbations with larger centre of mass (COM) excursions (Kuo and Zajac 1993; Henry et al. 1998) and higher frequency (above $1 \mathrm{~Hz}$ ) movements during stance (Creath et al. 2005). Recent research, however, has emphasized the use of knee 
movements as part of the response to multidirectional support-surface perturbations (Maki et al. 1994; Carpenter et al. 1999; Allum et al. 2002; Allum et al. 2008).

Difficulties with controlling balance and movement are symptoms of many different neurological conditions, including, e.g. Parkinson's disease, cerebellar ataxia, vestibular loss and stroke (Bloem et al. 2001; Carpenter et al. 2004; Bakker et al. 2006; Allum et al. 2008; Geurts et al. 2005; Horak et al. 2005). The mechanisms underlying postural instability in such patients have been studied using static or dynamic posturography. During posturography experiments, where subjects are exposed to sudden tilts of a supporting surface, a common characteristic of these diseases which has emerged is a lack of sufficient knee flexion concurring with falls (Bakker et al. 2006; Allum et al. 2008). It was suggested that this knee rigidity caused by insufficient active knee flexion underlies both the increased lateral sway and enhanced forward trunk pitch that were also observed in these patient groups (Bakker et al. 2006; Allum et al. 2008), and it is possible that this loss of knee flexibility contributes to their everyday movement problems. Another mechanism underlying knee rigidity is increased joint stiffness. This can be due to higher background knee muscle activity (Bakker et al. 2006) possibly as part of a changed movement strategy. For example, patients with total knee arthroplasty tend to change their strategies to control motion during walking, stair climbing, and balance corrections (Heller et al. 2001; Byrne et al. 2002; Gage et al. 2007), perhaps due to postoperative loss of knee proprioception and compensatory active stiffening of knee muscles. Such an increased intrinsic knee stiffness with aging may also contribute to the increased risk of falling seen in elderly populations, as does weakness or fatigue of knee muscles (Pavol et al. 2001; Bellew and Fenter 2006). Finally, reduced passive joint motion due to inelasticity of connective tissue and ligaments may also underlie knee stiffness causing movement rigidity.

One technique that may be employed to gain insights into the role of a specific joint in balance control is to artificially lock the joint, to such an extent that it is held rigid. For example, the importance of ankle motion to balance control was exemplified by studies of subjects fitted with ankle casts (Gurfinkel et al. 1974). Similarly, the contribution of motion about the hips and lumbo-sacral joints to balance control has been studied in healthy adults whose hips and lower trunk were artificially stiffened using a rigid corset (Grüneberg et al. 2004). Such experiments provided an empirical basis for correlating the reduction of joint motion due to either stiffness or movement rigidity with balance instabilities in certain disease states. For example, patients with a global absence of proprioceptive feedback due to a dorsal root ganglionopathy may develop an axial-stiffening strategy with both mechanisms that markedly resemble the pattern seen in healthy subjects fitted with trunk-stiffening corsets (Bloem et al. 2002).

In the present study, we have used this focal cast technique to further explore the role of knee motion by 'locking' the knees of healthy young adults with individually fitted, rigid knee casts on both legs. These subjects were exposed to multidirectional perturbations of quiet stance with and without casts. Our first specific goal was to determine to what extent, and how, reduced knee movement would impair balance control. We predicted that body motion would be similar to that of patients with balance problems associated with knee rigidity. For this reason we compared our results to those of a group of previously described patients with spinocerebellar ataxia (SCA) (Bakker et al. 2006). In these patients we noted that knee stiffening and active rigidity was associated with excessive lateral sway of the COM following medio-lateral perturbations presumably due to increased joint stiffness with increased muscle activity levels and to a lack of normal flexion of the "uphill" leg (opposite the direction of support surface tilt). In addition, excessive anterior-posterior sway of the COM was associated with excessive trunk flexion and changed pelvis motion (Bakker et al. 2006). Our second goal was to examine whether healthy subjects can initiate alternative, compensatory strategies to maintain effective balance control despite knee rigidity. Here, based on previous work (Grüneberg et al. 2004), we expected to identify an alternative balance-correcting strategy that included excessive forward pitching of the trunk, resembling the compensatory strategy adopted by SCA patients. It was an open question whether an alternative strategy would emerge to compensate for the lateral sway.

\section{Methods}

\section{Subjects}

We tested 18 young, healthy adults (nine men, age range 19-29 years) without orthopaedic or neurological disturbances as verified by a medical interview and routine clinical screening procedures (Allum and Carpenter 2005). All subjects gave written informed consent prior to the experiment. We conducted the experiments conform the standards of the Declaration of Helsinki. The Institutional Review Board of the University Hospital Basel approved the study.

All subjects were tested under two different conditions. One condition involved wearing individually fitted knee casts, constructed with Scotchcast/Softcast $\left(3 \mathrm{M}^{\mathrm{TM}}\right)$ on both legs, which reduced knee movement to a minimum (Fig. 1). The corsets did not restrict motion about the ankles or hips. In the other condition knee movement was without casts and therefore unrestricted. Half of the subjects were first 
tested while wearing the knee casts, the other half were first tested without the casts. There was a 6-8-week interval between the times of the two test conditions, in order to minimize a learning effect.

The results of these healthy subjects were compared to those of nine patients with SCA (seven men, age range 3260 years) that were tested using the same protocol (Bakker et al. 2006). Briefly, these patients had a genetically proven autosomal dominant spinocerebellar ataxia (SCA). One patient was diagnosed with SCA1, two with SCA2, two with SCA3, one had SCA6, and finally, three patients with SCA14. Their clinical presentation was dominated by cerebellar ataxia, with no or only minimal extracerebellar signs (such as spasticity or extrapyramidal features) that would affect balance, as verified during careful examination by two neurologists specialized in movement disorders, and supplemented with neuroimaging, EMG and vestibularocular reflex testing for most patients. Exclusion criteria were, loss of independent ambulation, severe visual disturbances or cognitive impairment. Three patients had pure cerebellar ataxia, without any extra-cerebellar features, and the remaining six patients had very mild extra-cerebellar features (for details see Bakker et al. 2006). To document that the responses of the young subjects without knee casts did not differ from those of middle-aged healthy subjects age-matched to the SCA subjects, comparison responses are provided in Figs. 1, 3, 4, 5.

\section{Protocol}

Balance control was examined with a dual-axis tilting platform, as described in previous studies (Carpenter et al. 1999; Allum et al. 2002; Bakker et al. 2006). The subject's feet were lightly strapped into heel guides fixed to the top surface of the platform. The heel guides were adjusted in the AP direction to ensure that the ankle joint axes were aligned with the pitch axis of the platform. Stance width was $14 \mathrm{~cm}$ (midheel to mid-heel) for all subjects. Handrails were located at each side of the platform $(40 \mathrm{~cm}$ from platform centre; $80 \mathrm{~cm}$ in height, adjustable to elbow height of subject). An assistant stood next to the platform to lend support in case of a fall.

Immediately before the start of the experiment, subjects were asked to assume their preferred stance position on the platform, arms hanging by the sides. Then anterior-posterior (AP) and medio-lateral (ML) ankle torques, derived from force movements recorded with strain gauge systems embedded into the surface of the platform, were sampled. These torque signals functioned as reference values for the preferred upright stance for that particular subject for the rest of the experiment.

All subjects were presented with 49 platform tilts in six different directions. Two directions were purely in the pitch plane $\left(0^{\circ}\right.$ toe down and $180^{\circ}$ toe up), the other four com- bined pitch and roll $\left(45^{\circ}\right.$ forward right $135^{\circ}$ backward right, $225^{\circ}$ backward left, and $315^{\circ}$ forward left). All perturbations were delivered with a constant velocity of $60 \%$ and constant amplitude of $7.5^{\circ}$. The very first trial was excluded from further analysis to reduce habituation effects in the data (Keshner et al. 1987). In the remaining trials, each of the six perturbation directions was randomly presented eight times. Halfway through the experiment, subjects were given a short-seated rest period in order to minimize fatigue.

Each perturbation was preceded by a random 5-20 s delay. During this delay, period visual feedback of the subject's AP and ML ankle torques (compared to the reference value measured directly before the experiment) was presented to the subject on a cross, embedded with rows of light-emitting diodes (LEDs) at four meters from the subject. This visual feedback was used to standardise prestimulus position across trials.

\section{Data collection}

Recordings of all biomechanical data commenced $100 \mathrm{~ms}$ prior to perturbation onset and lasted $1 \mathrm{~s}$. To collect full body kinematics, a three-dimensional optical tracking system with 21 infrared-emitting diodes (IREDs) (Optotrak, Northern Digital, Canada) was used. The three Optotrak cameras were placed approximately four meters in front of the subjects and sampled the IREDs signals at $64 \mathrm{~Hz}$. The IREDs were placed bilaterally on the following anatomical landmarks: frontally at the level of the lateral malleolus; on the centre of the patella; frontally at the level of the greater trochanter, on the anterior superior iliac spine; processus styloideus; frontally in the middle of the elbow axis; on the acromion, and on the sides of a head-band placed just above the ears. In addition, one diode was placed on the angulus sterni and one on the chin. For reference, three IREDs were placed at the front corners and on the left side of the platform to define pitch and roll movements of the platform. All subjects wore tight-fitting shorts and vests to prevent marker movements. Knee flexion was measured as the angle subtended between the markers on the hip at the level of the greater trochanter, on the cast over the patella, and at the ankle joint.

Data analysis

Following analog to digital data conversion, all biomechanical signals (ankle torques and platform angles) were averaged off-line across each perturbation direction. Zero latency was defined as the onset of platform rotation. Subject averages were pooled within both conditions to produce population averages for a single direction. 
Position data were digitally filtered at $16 \mathrm{~Hz}$ using a zero phase shift fourth order Butterworth filter. Our main outcome measure, total body COM displacement was calculated separately for the anterior-posterior (AP), medio-lateral (ML) and vertical directions using an 11-body segment adaptation of a 14-segment model (Winter 1990). Each arm was modelled as two units, (upper and lower arms) and two trunk segments (trunk, pelvis) were used instead of four. Absolute rotation angles of the planes defined by trunk and pelvis body segments and the platform surface were defined using three or four markers on these segments. Stimulus-induced changes were calculated with respect to averaged angles over a pretrigger time interval of $90 \mathrm{~ms}$ ending $10 \mathrm{~ms}$ prior to the stimulus onset. Our main outcome measure, the area under the curve of COM displacement was calculated from the onset of platform movement $(0 \mathrm{~ms})$ to $800 \mathrm{~ms}$ later.

To explore angle changes between body segments, we calculated angle changes that were calculated at two predefined times: 300 and $800 \mathrm{~ms}$. The measurement at $300 \mathrm{~ms}$ was chosen to quantify the impact of balance-correcting responses on kinematics. For arm movements, a later time $400 \mathrm{~ms}$, coinciding with peak arm abduction (see Bakker et al. 2006) was chosen. The measurement at $800 \mathrm{~ms}$ marked the end of our recording period where subjects approached a stable end position.

\section{Statistical analyses}

Our primary analyses concerned between-condition comparisons for rigidity effects caused by the casts. After ascertaining that the data values were normally distributed, differences between different perturbation directions and between the two cast conditions were tested using a repeated measures ANOVA model (cast condition $\times$ direction). Significant $(P<0.05)$ main cast and interaction effects were further explored using post-hoc paired Student's $t$-tests. A secondary analysis involved a comparison with previously published results on SCA patients (Bakker et al. 2006) and the subjects of the present study with knee casts. Differences were first tested for population differences with a repeated measures ANOVA model and then post-hoc independent $t$ tests were used to compare results in each direction. The significance level of all the post-hoc test results was set to account for the effect of comparing multiple measurements at once (Bonferroni correction).

\section{Results}

Effectiveness of knee casts in reducing knee flexion

Figure 1 illustrates mean population knee flexion traces in young controls with and without the knee casts, as well as those from middle-aged controls and SCA patients. These traces show that knee flexion was effectively prevented by the casts, typically amounting $1^{\circ}$ or less. Figure 1 (right panels) illustrates the average knee angle changes, as measured at $800 \mathrm{~ms}$ after stimulus onset. A main effect of knee casts on knee angles was observed for both the uphill $(F(1,17)=41.2 ; P<0.001)$ and downhill $(F(1,17)=24$; $P<0.001$ ) knees. (The uphill knee is the left knee when the tilt is right-side down.) For the uphill knee, flexion with casts was significantly less for all tilt directions (Fig. 1 right), and for the downhill knee the knee flexion was less for all forward tilt directions (Fig. 1 right). Knee flexion of the downhill knee for backward tilt directions was less than $1^{\circ}$ even without casts as the knee is normally locked into extension. For forward tilts, there is flexion in the downhill knee-see Fig. 1, left. Figure 1 also shows that knee flexion (or lack thereof) in the downhill knee was similar for normals with casts and SCA patients $(P>0.05)$. However, for the uphill knee, knee flexion in patients was slightly larger without reaching significance.

\section{Effects on centre of mass movements}

Bilaterally fitted knee casts increased the anterior-posterior (AP) displacement of the COM (Fig. 2). This was confirmed by analysis of the area under the COM curve between 0 and $800 \mathrm{~ms}$ after stimulus onset $(F(1,17)=7.3$, $P<0.02)$. Post-hoc tests revealed that this difference was only significant for backward directions with and without roll ( $P<0.01$; see Fig. 2, upper right).

The knee cast reduced the lowering of the COM (Fig. 2), but only for forward directed perturbations (Fig. 2 lower right). We found a significant condition by direction interaction $(F(1,85)=7.6 ; \quad P<0.01)$, with a significantly decreased lowering of the COM for forward directions only $(P<0.05)$. No effect of knee casts on medial lateral (ML) COM displacement was observed.

Figure 2 also compares the changes in COM traces with the casts and illustrates those of SCA patients (data reproduced from Bakker et al. 2006). The amplitudes of AP COM displacements of SCA patients tended to be greater than those induced by the casts in normals direction by group interaction, $F(1,85)=6.3 ; P=0.01$ ), particularly in the backward (and roll) directions, but post-hoc tests revealed no differences due to the large variances recorded for SCA patients. The amplitude of vertical COM displacements of SCA patients was not significantly different from those of normals with casts for forward directions $(P>0.05)$. ML COM displacements were greater for SCA patients than for normals with casts $(F(5,40)=3.7 ; P<0.01$, a cast by direction effect). 
Fig. 1 Biomechanical responses of the knee to platform tilts into different directions. In the left panels, the course of the knee angle after a forward right $\left(45^{\circ}\right)$ and backward-right $\left(135^{\circ}\right)$ platform tilt is shown. The traces represent the mean response of 18 young $(\mathrm{YN})$ subjects with and without a double knee cast, nine spino cerebellar ataxia (SCA) patients and a set of age-matched middle-aged (MA) controls (SCA and age-matched control data from Bakker et al. 2006). The dotted line at $0 \mathrm{~ms}$ indicates the onset of support surface tilt. In the right panels the changes in the knee angle at $800 \mathrm{~ms}$ are shown for the uphill and downhill knees. The columns represent the mean change and the bars over the columns standard error of the mean for the two cast conditions and for SCA patients. Differences between the normal subject means are indicated with and without casts by asterisk $(*)$ symbols
Version 29 of 17.11.2007

Uphill (Left) knee angle reponses, 135"

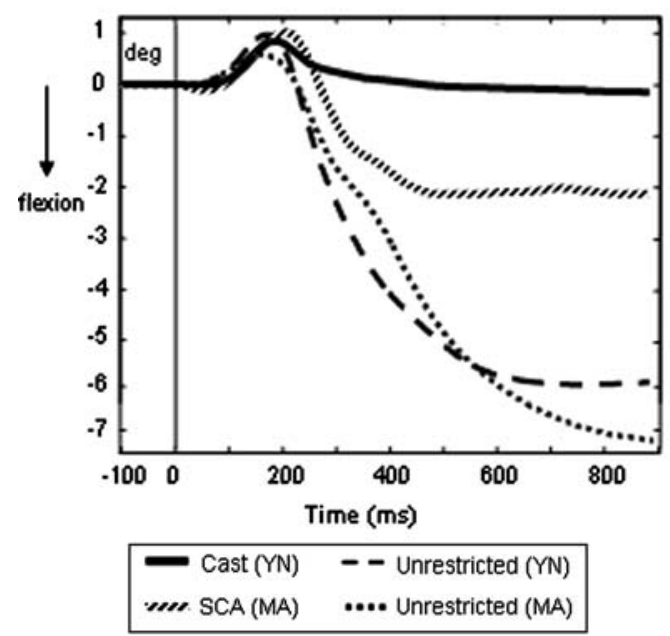

Downhill (right) knee angle responses, 45*

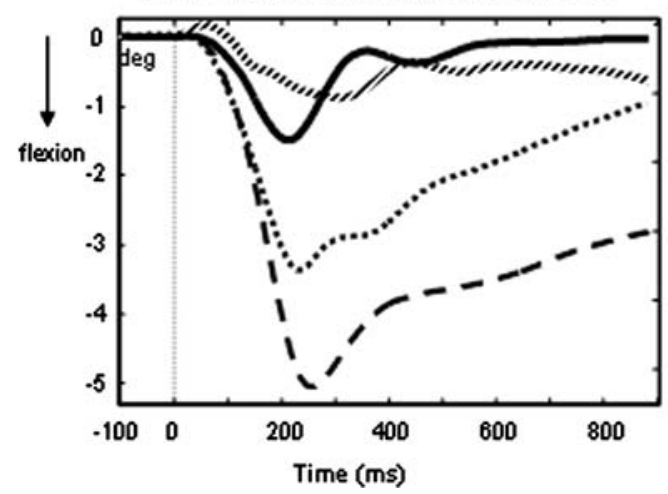

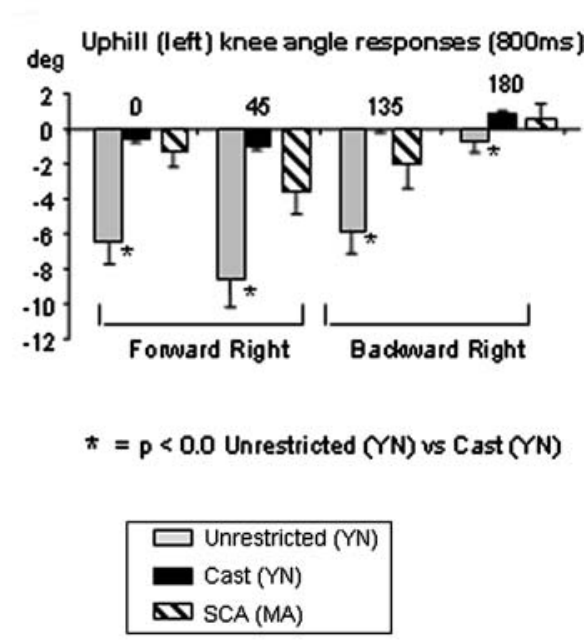

$\star=p<0.0$ Unrestricted (YN) vs Cast (YN)

\section{Knee angles}

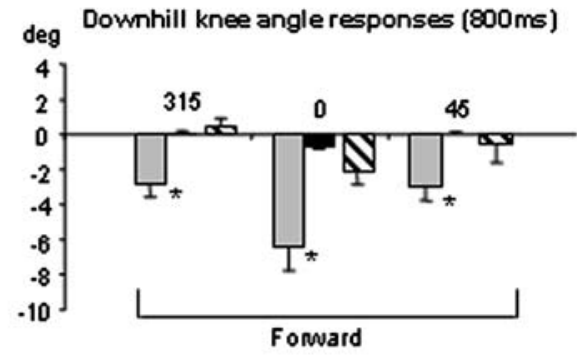

Movements at ankle, hip and lumbrosacral joints

In the following description of the results joint movements in the sagittal plane are described first, and then joint movements in the frontal plane.

Application of the casts led to changes in both uphill and downhill ankle angles at both 300 and (as shown in Fig. 3) at $800 \mathrm{~ms}(F(1,17)$ ranged $40.9-47.8 ; P<0.001)$. The ankle angle displacements for those with casts were not different from those seen in SCA patients $(P>0.05)$. Examples of these changes are illustrated in Fig. 3 for the tilt direction of $135^{\circ}$, where ankle motion was smaller in both SCA patients and controls with casts compared to controls without casts. This effect can be observed in Fig. 3 for all backward tilt directions $(P<0.01)$. For forward directions the ankle plantar flexion was greater with casts (Fig. 3). There were no differences in ankle angle changes between SCA patients and controls fitted with casts (no cast effect or cast by direction interaction).

Pelvis pitch rotation changed direction when casts were placed on the legs (Fig. 4 left). Specifically, the pelvis always pitched forward in normals with casts, and very similar observations were made for SCA patients. This effect was seen for all perturbation directions (Fig. 4 right), and was confirmed statistically by a main Group effect at both the 300 and $800-\mathrm{ms}$ measurement times $(F(1,17)=31.4$ and $25.0 ; P<0.001)$. Post hoc analyses showed no significant differences for displacements in normals with casts and SCA patients $(P>0.05)$, but a significant difference between these groups and young controls without casts $(P<0.05$.

Hip flexion angles were less strongly influenced by the knee casts than the ankle angles. There was a main effect of the cast on the uphill hip angle at both 300 and $800 \mathrm{~ms}$ $(F(1,17)=27.5 ; P<0.001)$. For all tilt directions with a roll component, uphill hip flexion angle changes were less with the casts $(P<0.01)$. In the downhill hip, hip angle changes with the casts were weaker at $300 \mathrm{~ms}$ $(F(1,17)=6.1 ; P=0.024)$ and at $800 \mathrm{~ms}$, for which only a cast by direction interaction was observed $(F(1,85)=9.6$; $P<0.01)$. For the backward tilt directions increased hip flexion was observed in the downhill leg with the casts.

The effect of the casts on trunk pitch was weak. Only a cast by direction effect was observed with greater forward pitch at 
Fig. 2 The effects of the platform perturbations on the anterior-posterior (AP), vertical and mediolateral COM displacements to different directions of tilt. The layout of the figures is identical to Fig. 1

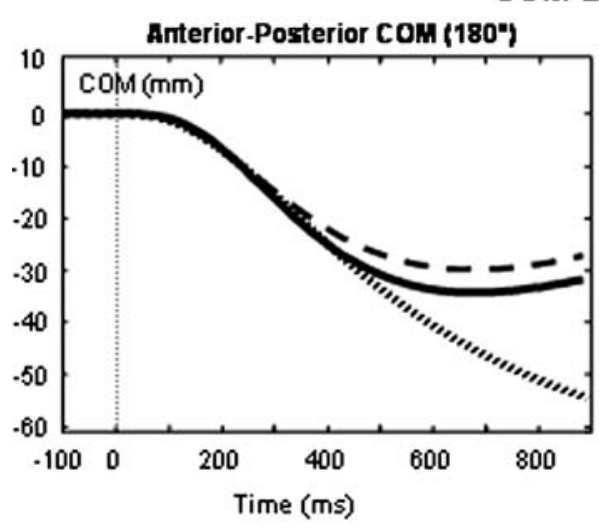

COM Displacements

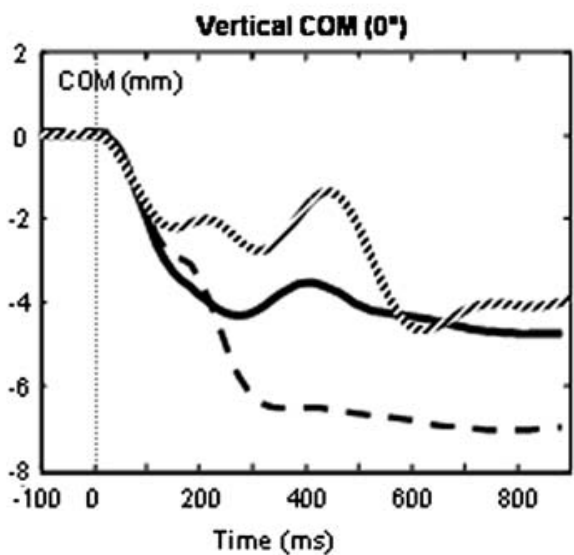

$x=p<0.01 ; \#=p<0.05$ Unrestricted $(\gamma N)$ vs $\operatorname{Cast}(\gamma N)$

(mmsec)

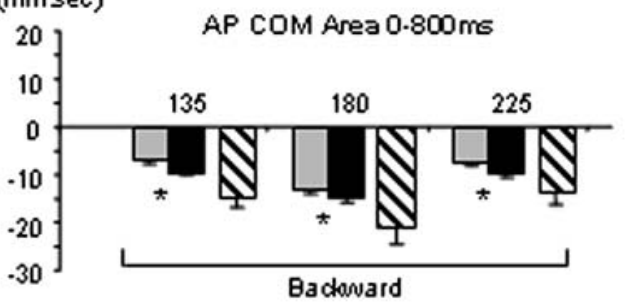

(mmsec) Vertical COM Area $0.800 \mathrm{~ms}$
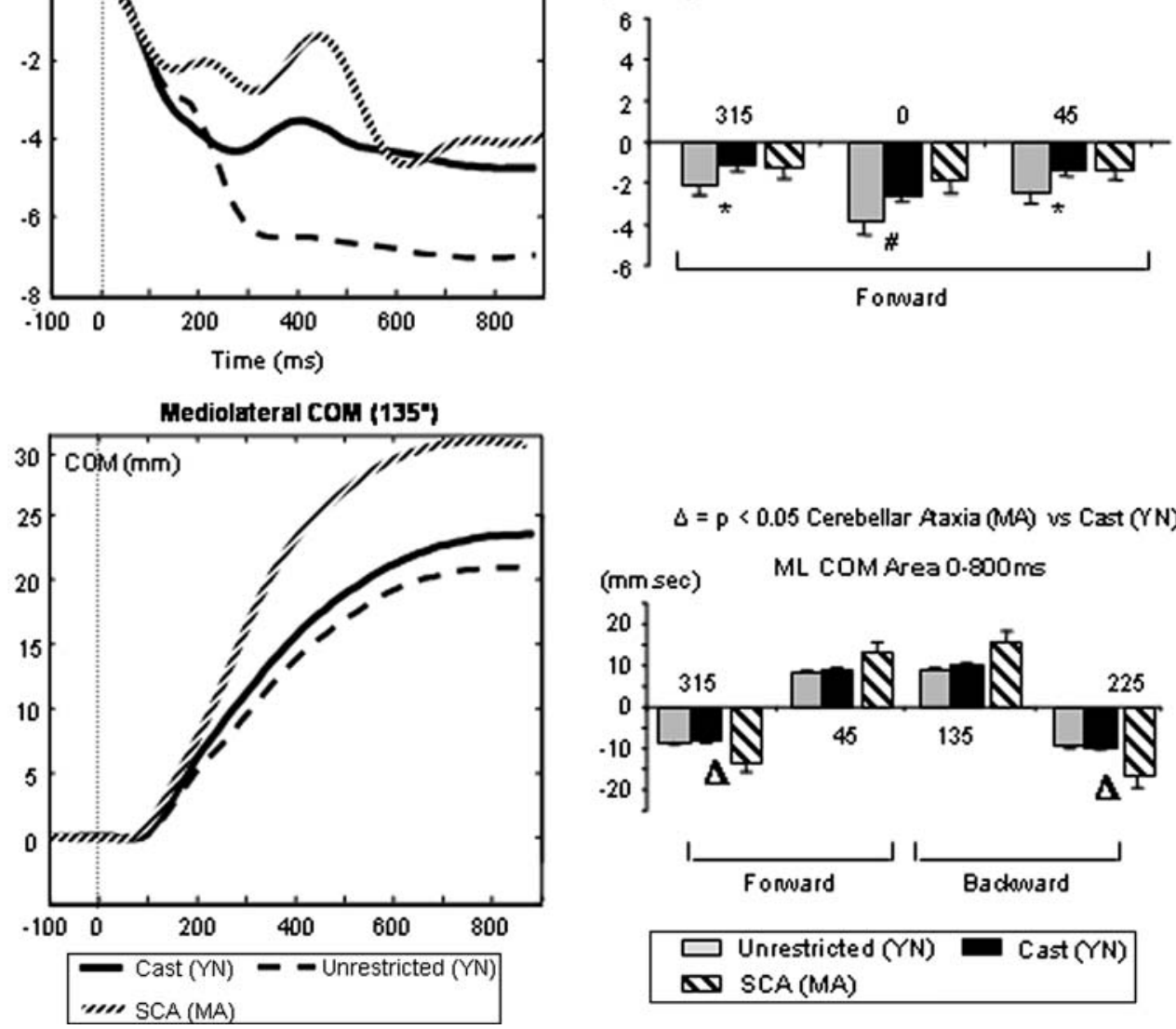

$\Delta=p<0.05$ Cerebellar Aaxia (MA) vs Cast $(\gamma N)$

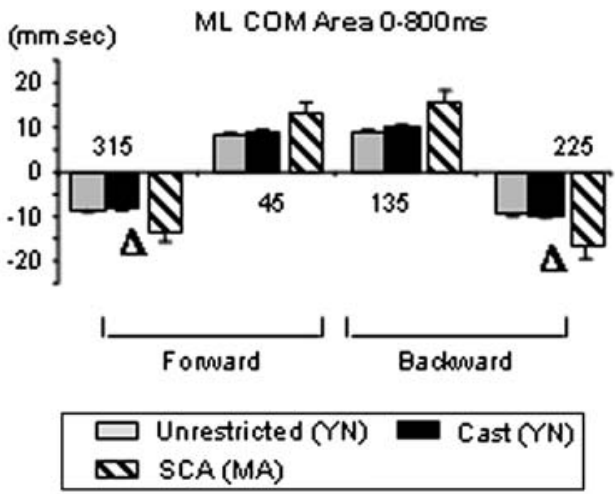

$800 \mathrm{~ms}$ when the support surface was tilted backwards $(P<0.05)$. SCA patients showed similar forward pitch for backward tilt as controls with casts but greater forward pitch at $800 \mathrm{~ms}$ for all directions of forward tilt (Fig. 5 right).

Lateral movements of the pelvis and arms

Pelvis roll movements at $800 \mathrm{~ms}$ were increased with the knee casts (lower part Fig. 4). Across all directions there was a cast by direction effect $(F(1,85)=4.5 ; P<0.01)$. This effect was greater when the pure pitch directions were excluded from the analysis, because post-hoc analyses revealed increased pelvis roll for all roll directions except backwards right $\left(135^{\circ}\right)$. Pelvis roll tended to be greater in SCA patients compared to healthy controls with casts (Fig. 4) but no significant differences were observed. Trunk roll was not different with and without the knee casts $(P>0.05)$. 
Fig. 3 Ankle angle responses to platform tilts into different directions. See legend to Fig. 1 for details
Casts led to increased arm abduction in the uphill but not in the downhill arm $(F(1,85)=8.5 ; P<0.01)$. Post-hoc analysis revealed significant differences for the left but not the right arm, when this was the uphill arm (Fig. 5). The amplitudes of arm movement when wearing casts were significantly less than those of SCA patients for the uphill arm $(F(1,85)=6.8 ; \quad P<0.05)$. Downhill arm abduction responses did not differ between subjects with knee casts and SCA patients.

\section{Discussion}

The young subjects in this study were capable of adapting their movement strategies when forced to correct for tilts of the support surface with the knees held in locked extended position. The AP COM displacement was increased, the vertical displacement reduced and the ML displacement was unaltered when subjects wore the casts. Explorative analyses showed that the compensatory strategy in the pitch plane involved a change to forward pelvis rotation and alterations in hip and ankle joint movement strategies. In the roll plane, arm movements were increased and hip flexion in the uphill leg was decreased. The very fact that control subjects needed to adopt different strategies to counter
Ankle Angles
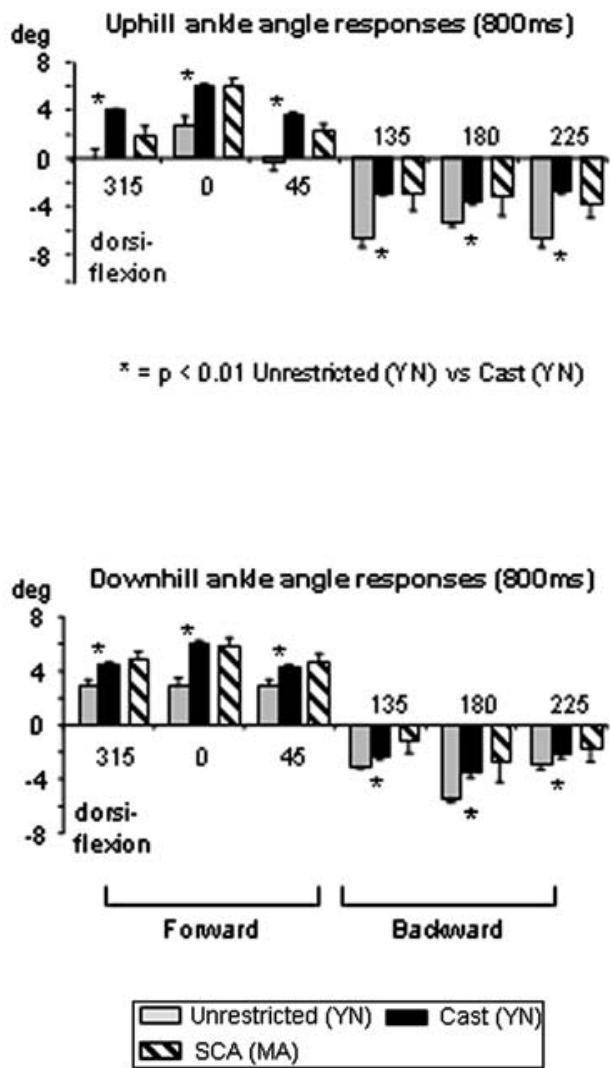

the effect of reduced knee flexion in order to retain balance, underscores that knee movements are an integral part of the normal balance-correcting synergy for correcting tilt of the support surfaces.

Figure 6 provides a summary of our findings for joints in the pitch plane, and also compares at each joint the changed strategy in normals with casts and that used by SCA patients. The schematic representation shows the strategy adopted for a backward roll and a forward roll tilt of the support surface. Generally, the strategies are similar for controls with casts and SCA patients following backward and roll perturbations, but not following forward and roll perturbations. In controls with knee casts, ankle dorsi-flexion in both the uphill and downhill ankles was less for backward perturbations and plantar-flexion was greater for forward perturbations. A similar action was observed in SCA patients. Hip flexion was also differentially modulated for backward and forward roll tilts in subjects with knee casts. For backward roll tilts, the uphill leg had greater flexion than the downhill leg when knee action was unrestricted. This action was reversed with the casts, that is, less flexion occurred at the hip in the uphill leg. This hip action is unlike that of SCA patients. A similar disparity was observed for the uphill leg after forward roll tilts for which little flexion occurred with the casts. Together with the 
Fig. 4 Biomechanical responses of the pelvis to multi-directional platform perturbations. Traces of the time course of the pelvis pitch and roll angles for the unrestricted and cast conditions and for SCA patients and age-matched controls after a platform tilt are shown on the left. Mean changes in the angle in both conditions are shown on the right for all tilt directions

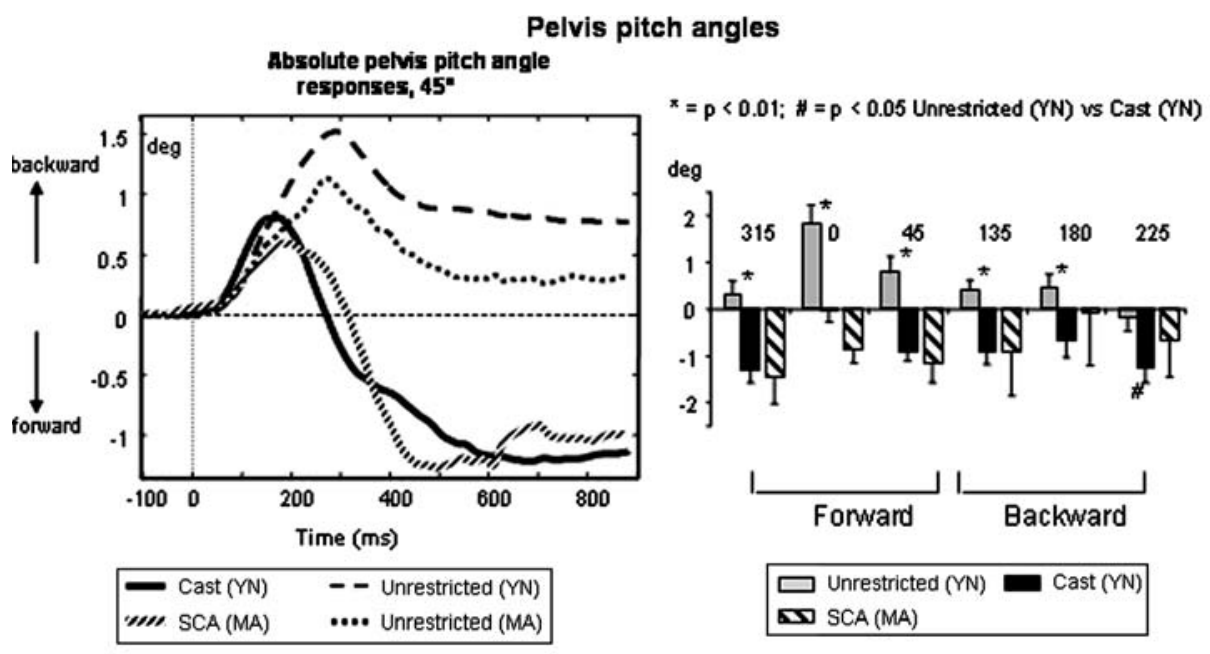

Pelvis roll angles
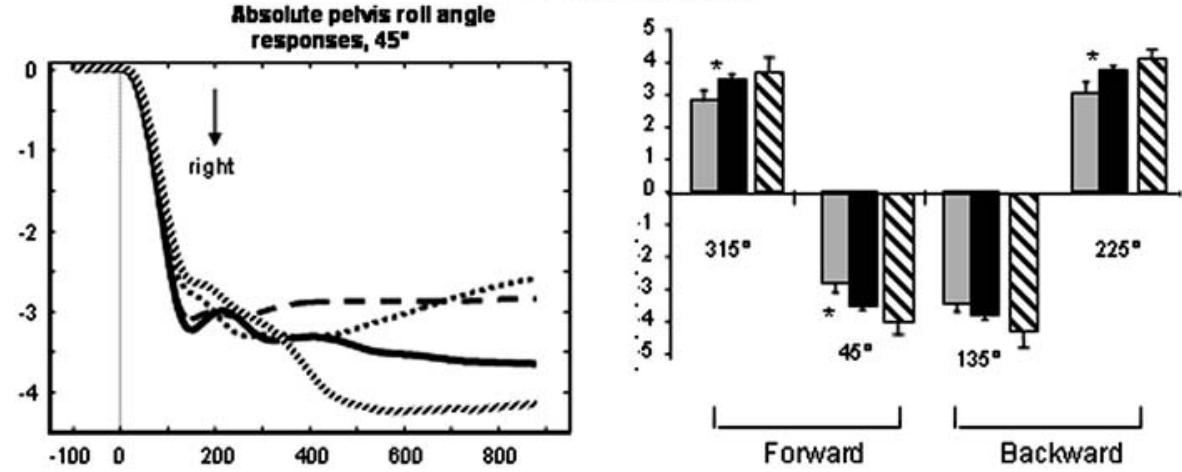

increased arm abduction, the changes in hip movements with the casts could underlie the ability of normal subjects to cope with lateral movements with the knee casts.

The most obvious effect of absent knee movement was on the vertical and AP COM movement. With knee movements unrestricted, the vertical COM height is lowered when the knees bend in response to forwards tilt. Application of the knee casts reduced this lowering of the COM. For backward directed tilts, the posterior displacement of the COM was greater with knee casts. Interestingly, the knee casts did not affect the lateral movement of the COM. Our working hypothesis was that the rigidity of the knees might account for the lateral and backward-AP instability of SCA patients (Bakker et al. 2006). Our present findings show that this hypothesis can only be valid provided that healthy subjects do not adopt different compensating strategies to overcome the effect of stiffened knees. In other words, if healthy subjects with stiffened knees respond to the tilt perturbations in a manner similar to that of SCA patients. The similarity of the changes in vertical and AP COM movements in the subjects with casts and SCA patients suggest that this is indeed the case for pitch components of the tilt. The current findings indicate that the knee casts did not affect the lateral displacement of the COM because the control subjects adopted a different, but more effective compensatory strategy. Thus, it is still possible that knee joint stiffness or lack of knee flexion underlie the deficit in lateral sway control in SCA subjects. This study was not able to provide support for this hypothesis. Here, we have used results from SCA patients in an attempt to compare effects seen with stiffened knees in healthy controls with the balance deficits of these patients. The effect of the compensatory strategies adopted by healthy controls would be an issue to be raised regardless of the comparison patient group chosen.

Our findings also shed new light on the adaptive strategies developed by SCA patients (and possibly other patient groups with balance problems), who have pathologically reduced knee flexion as a "stiffening strategy" to reduce multilink interactions and thereby facilitate motor control (Morton and Bastian 2004; Bakker et al. 2006). While such knee stiffening may be advantageous during gait, it interferes with the necessary rapid knee movements that are required to absorb the impact of "toes-down" and laterally directed platform tilts with opposite side "toe-up" components. As described previously (Bakker et al. 2006), SCA patients compensated for this lack of knee motion by resorting to increased forward flexion of the trunk, thereby mov- 
Fig. 5 Biomechanical responses of the trunk and uphill arm to multi-directional platform perturbations. Traces of the time course of the trunk angle and uphill (left) arm for the unrestricted and cast conditions and for SCA patients and age-matched controls after a platform tilt are shown on the left. Mean changes in the angles for the same conditions are shown on the right for all tilt directions

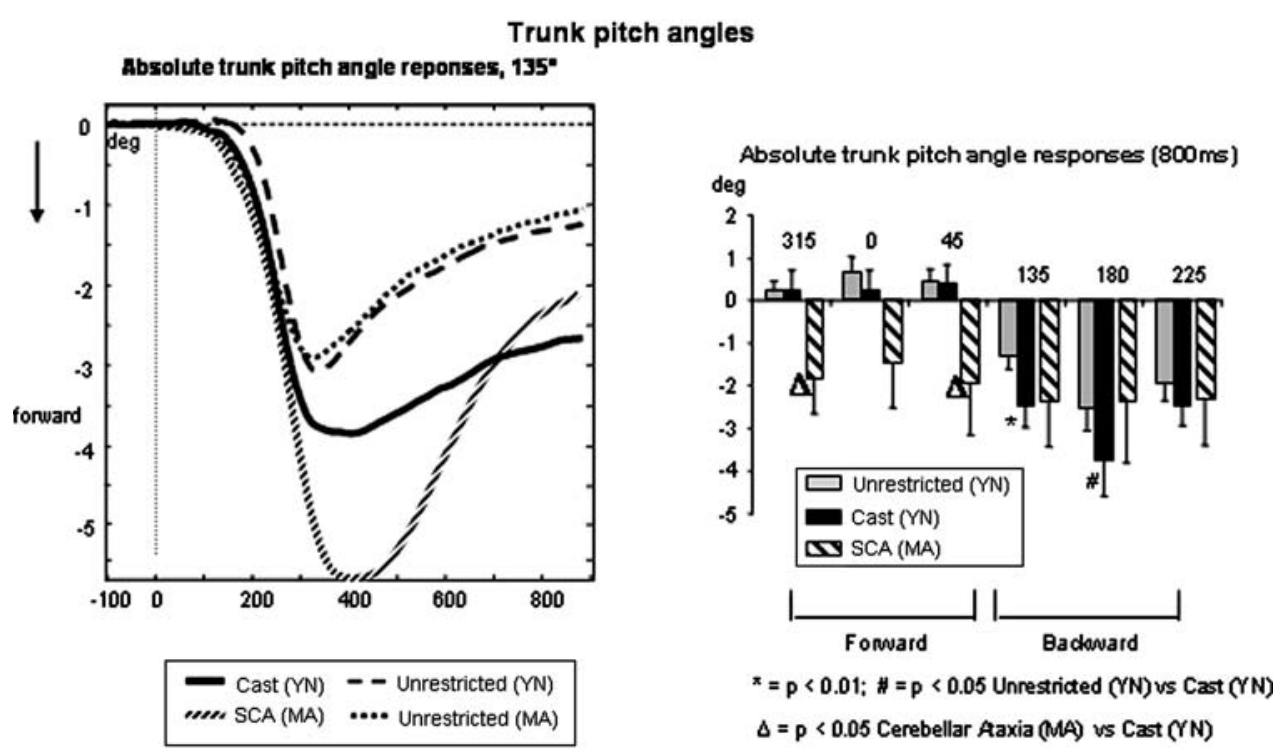

Uphill arm abduction angles

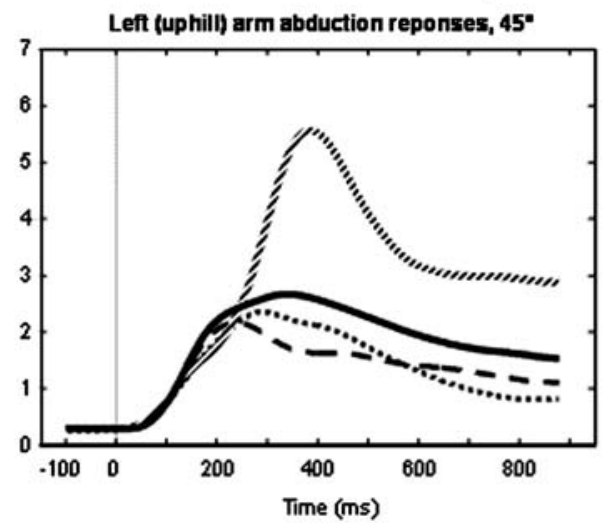

ing the COM forward and reducing the risk of a possible backward fall. However, this forward trunk flexion is present in SCA patients irrespective of perturbation direction, unlike the normals with knee casts who also increased their trunk flexion, but only for backward-directed perturbations, where such compensation is functional to reduce backward sway. SCA patients seem to have selected trunk flexion as their "default" strategy, even though this goes at the expense of increased forward sway for forward-directed perturbations when such trunk flexion is not required and possibly harmful. One possible explanation could be fear of falls, which is common in patients with SCA (van de Warrenburg 2005), particularly for backward-directed falls. The price paid could be a higher incidence of forward falls in daily life, which are indeed not uncommon in SCA patients (van de Warrenburg 2005). Knee stiffness may further contribute to forward falls by interfering with the rapid knee flexion movements that are required to avoid tripping over obstacles (Pijnappels et al. 2005). Indeed, patients with bilateral total knee arthroplasty have a reduced ability to negotiate obstacles, and this increases their risk of tripping (Mauer et al. 2005).

A major change in synergy observed with the casts was an increased forward rotation of the pelvis. This may be the result of an attempt by balance-control centres to simplify the control strategy by "locking" the pelvis to the trunk effectively creating a single more massive trunk unit. Combined with an increased trunk forward pitch for backward tilts, this forward rotation of the pelvis can be interpreted as an attempt by balance-control centres to place the COM more forward. A similar action was observed in SCA patients. However, this strategy change, as in the SCA patients, appears to be unsuitable to providing improved stability. In fact, the pelvis horizontal position ended up being displaced more backwards with the casts (not reported in the results) just as in the SCA patients (Bakker et al. 2006) with a similar effect on posterior COM position.

One of the limitations of our comparisons with SCA patients is that the ages of the patients (average 49 years, see Bakker et al. 2006) were higher than those of the young 

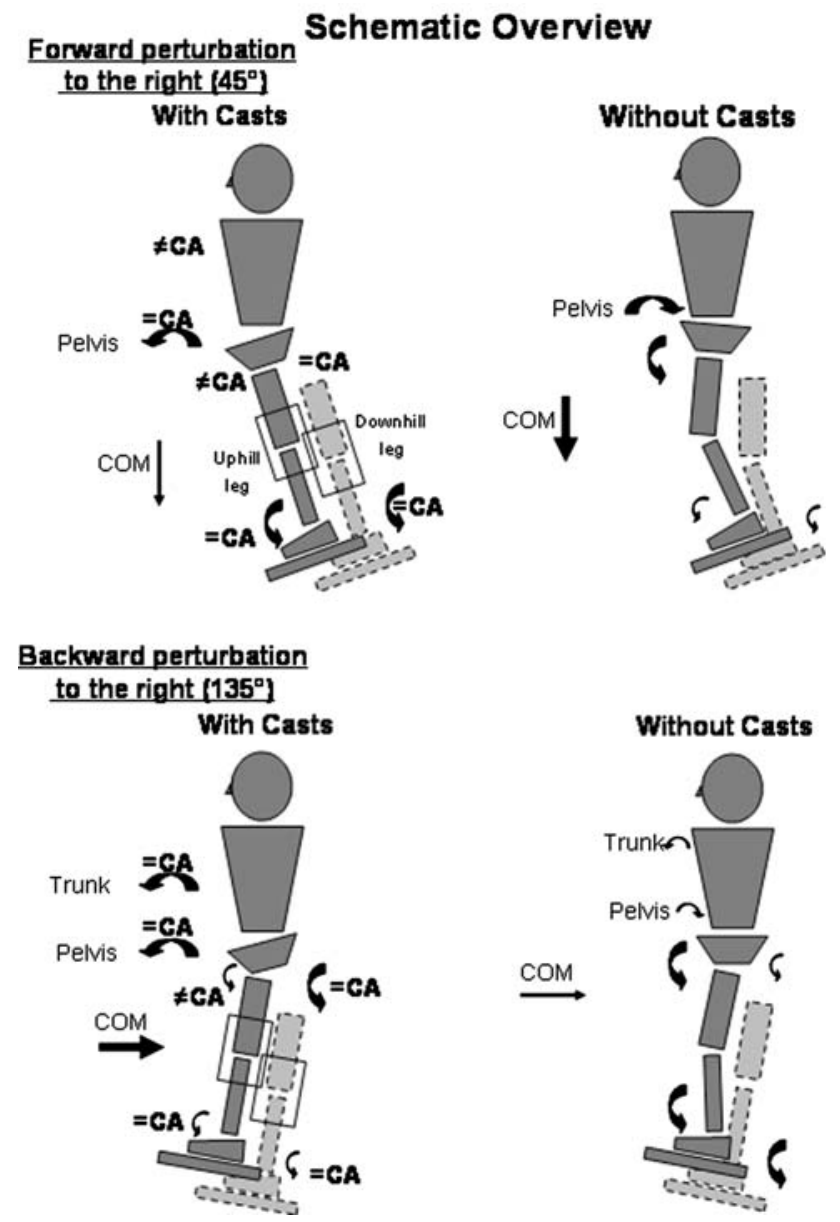

Fig. 6 Schematic overview of pitch-directed biomechanical responses seen in the two conditions for forward-right $\left(45^{\circ}\right)$ and backward-right $\left(135^{\circ}\right.$ ) perturbations, at $800 \mathrm{~ms}$ after platform perturbation onset (when a more or less stable end position had been reached). The knee casts are indicated by rectangles drawn around the knees. Changes in the different trunk and leg angles of the uphill and downhill leg and changes in COM position are indicated by arrows. The size of the arrows represents the amount of angular change with respect to the joint angle prior to the perturbation. If the motion was similar in SCA patients the symbol " = CA" is used, if not " $\neq \mathrm{CA} "$. The symbols "=CA" or " $\neq \mathrm{CA} "$ thus indicate changed strategies of control with respect to those of SCA patients

adults fitted with knee casts in this study. The major effect of age for perturbations comparable to the ones used in the present study (Allum et al. 2002) is on lateral stiffness of the trunk, rather than on anterior-posterior stiffness of the trunk. However, the study by Allum et al. (2002) did not specifically look at knee angle and pelvis angle responses, which tended to be less in the middle-aged compared to the young (see Figs. 1, 4). Thus, the trends we observed for these responses to be stiffer in the middle-aged might represent a general stiffening with age in other joints too. Nonetheless, we would not expect differences in AP displacements if we had used middle-aged normals with casts as a model for studying the posterior instability of
SCA patients on backward tilt. Evidence supporting this assumption is provided in the traces of Figs. 1, 3, 4 and 5. Here, the responses of the young normals of the current study may be compared with those of another group of middle-aged control subjects age-matched to SCA patients (data from Bakker et al. 2006). The traces for unrestricted knee movements in response to the perturbations are generally similar for both normal control groups but very different from the responses of the young normals with knee casts. We assume that adding knee casts to a middle-aged normal population would have yielded similar effects to those described in the current research.

This study provided a description of kinematic responses engendered by stiffening the knees, which may be useful in understanding not only various pathologies such as SCA, but also osteoarthritis. Bilateral knee stiffening seems, on the basis of the current results, to provide an explanation for the AP but not for the lateral instability of SCA patients. Nonetheless, the changes in pitch control and the fact that young subjects fitted with casts efficiently learned to compensate for the lack of knee responses provides supporting evidence for our conclusion that knee movements are important in balance control. Future studies should concentrate on finding other ways to mimic the deficit in lateral balance-control in SCA patients. One such way may be the use of more extensive casts-for example, to simultaneously stiffen the knees and hips. Indeed, a previous study showed surprisingly little changes in overall stability in subjects fitted with a rigid corset that only stiffened the hips and lower trunk despite increased trunk velocities. More dramatic alterations emerged when a more extensive cast was used that also stiffened the upper trunk (Gruneberg et al. 2004). Another interesting option would be a temporary introduction and subsequent removal of knee stiffness rather than restricting the motion entirely, as this might provide a model to document adaptive motor control strategies (Noble and Prentice 2006). Insights from such studies could help to disentangle primary pathophysiological processes from compensatory adaptive strategies, and thereby help to shape rehabilitation strategies focused on improving knee motion or strength (see, e.g. Lord et al. 2005) in order to prevent falls.

Acknowledgments The research was supported by Swiss National Science Fund grant 3100A0-104212/1 to JHJ Allum. L.B. Oude Nijhuis was supported by a research grant (No. 00099) from the Radboud University Nijmegen Medical Centre, The Netherlands. M. Bakker was supported by a reseach grant from the Internationaal Parkinson Fonds.

\section{References}

Allum JHJ, Carpenter MG (2005) A speedy solution for balance and gait analysis: angular velocity measured at the centre of mass. Curr Opin Neurol 18:15-21 
Allum JHJ, Carpenter MG, Honegger F, Adkin AL, Bloem BR (2002) Age-dependent variations in the directional sensitivity of balance corrections and compensatory arm movements in man. J Physiol 542:643-663

Allum JHJ, Nijhuis Oude LB, Carpenter MG (2008) Difference in coding provided by proprioceptive and vestibular sensory signals may contribute to lateral instability in vestibular loss subjects. Exp Brain Res 184:391-410

Bakker M, Allum JHJ, Visser JE, et al (2006) Postural responses to multi-directional stance perturbations in cerebellar ataxia. Exp Neurol 202:21-35

Bellew W, Fenter PC (2006) Control of balance differs after knee or ankle fatigue in older women. Arch Phys Med Rehabil 87:14861489

Bloem BR, Allum JHJ, Carpenter MG (2002) Triggering of balance corrections and compensatory strategies in patients with total leg proprioceptive loss. Exp Brain Res 142:91-107

Bloem BR, van Vugt JPP, Beckley DJ (2001) Postural instability and falls in Parkinson's disease. Adv Neurol 87:209-223

Byrne JM, Gage WH, Prentice SD (2002) Bilateral lower limb strategies used during a step-up task in individuals who have undergone unilateral total knee arthroplasty. Clin Biomechanics 17:580-585

Carpenter MG, Allum JH, Honegger F (1999) Directional sensitivity of stretch reflexes and balance corrections for normal subjects in the roll and pitch planes. Exp Brain Res 129:93-113

Carpenter MG, Allum JH, Honegger F, Adkin AL, Bloem BR (2004) Postural abnormalities to multidirectional stance perturbations in Parkinson's disease. J Neurol Neurosurg Psychiatry 75:12451254

Creath R, Kiemel T, Horak F, Peterka R, Jeka J (2005) A unified view of quiet and perturbed stance: simultaneous co-existing excitable modes. Neurosci Lett 377:75-80

Fitzpatrick RC, Rogers DK, McCloskey DI (1994) Stable human standing with lower-limb muscle afferents providing the only sensory input. J Physiol 480:395-403

Fitzpatrick RC, Taylor JL, McCloskey DI (1992) Ankle stiffness of standing humans in response to imperceptible perturbation: reflex and task-dependent components. J Physiol 454:533-547

Gage WH, Winter DA, Frank JS (2007) Organization of postural responses following a rotational support-surface perturbation, after TKA: Sagittal plane rotations. Posture Gait 25:112-120

Gatev P, Thomas S, Kepple T, Hallett M (1999) Feedforward ankle strategy of balance during quiet stance in adults. J Physiol 514:915-928

Geurts AC, de Haart M, van Nes IJ, Duysens J (2005) A review of standing balance recovery from stroke. Gait Posture 22:267-281

Grüneberg C, Bloem BR, Honegger F, Allum JHJ (2004) The influence of artificially increased hip and trunk stiffness on balance control in man. Exp Brain Res 157:472-485
Gurfinkel VS, Lipshits MI, Popov KE (1974) Is the stretch reflex a basic mechanism in the system of regulation of human vertical posture? Biofizika 19:744-748

Heller MO, Bergmann G, Deuretzbacher G, Dürselen L, Pohl M, Claes L, Haas NP, Duda GN (2001) Musculo-skeletal loading conditions at the hip during walking and stair climbing. $\mathrm{J}$ Biomech 34:883-893

Henry SM, Fung J, Horak FB (1998) EMG responses to maintain stance during multidirectional surface translations. J Neurophysiol 80:1939-1950

Horak FB, Dimitrova D, Nutt JG (2005) Direction specific instability in subjects with Parkinson's disease. Exp Neurol 193:504-521

Keshner EA, Allum JHJ, Pfaltz CR (1987) Postural coactivation and adaptation in the sway stabilizing responses of normals and patients with bilateral vestibular deficit. Exp Brain Res 69:77-92

Kuo AD, Zajac FE (1993) Human standing posture: multi-joint movement strategies based on biomechanical constraints. Prog Brain Res 97:349-358

Lord SR, Tiedemann A, Chapman K, Munro B, Murray SM, Gerontology M, Ther GR, Sherrington C (2005) The effect of an individualized fall prevention program on fall risk and falls in older people: a randomized, controlled trial. J Am Geriatr Soc 53:12961304

Maki BE, McIlroy WE, Perry SO (1994) Compensatory responses to multidirectional perturbations. In: Taguchi K, Igarashi M, Mori S (eds) Vestibular and neural front. Elsevier, Amsterdam, pp 437 440

Mauer AC, Draganich LF, Pandya N, Hofer J, Piotrowski GA (2005) Bilateral total knee arthroplasty increases the propensity to trip on an obstacle. Clin Orthop Relat Res 433:160-165

Morton SM, Bastian AJ (2004) Cerebellar control of balance and locomotion. Neuroscientist 10:247-259

Nashner LM, McCollum G (1985) The organization of human postural movements: a formal basis and experimental synthesis. Behav Brain Sci 8:135-172

Noble JW, Prentice SD (2006) Adaptation to unilateral change in lower limb mechanical properties during human walking. Exp Brain Res 169:482-495

Pavol MJ, Owings TM, Foley KT, Grabiner MD (2001) Mechanisms leading to a fall from an induced trip in healthy older adults. J Gerontol A Biol Sci Med Sci 56:M428-M437

Pijnappels M, Bobbert MF, van Dieen JH (2005) How early reactions in the support limb contribute to balance recovery after tripping. J Biomech 38:627-634

van de Warrenburg BP, Steijns JA, Munneke M, Kremer BP, Bloem BR (2005) Falls in degenerative cerebellar ataxias. Mov Disord 20:497-500

Winter D (1990) Biomechanics and rotor control of human measurement, 2nd edn. Wiley, New York 